\title{
Emotional Laour in Teaching Secondary Physical Education
}

\author{
Ye Hoon Lee \\ Department of Health, Physical Education, and Recreation, University of North Alabama, PO box 5073, Florence, AL, USA \\ E-mail: ylee6@una.edu \\ Hyungil Harry Kwon (Corresponding author) \\ Department of Physical Education, Chung-Ang University, \#202, bldg. 305, Chung-Ang Univ., 84, Heukseok-ro, Dongjak-gu, Seoul, South Korea \\ E-mail: hkwon@cau.ac.kr \\ Hwajung Oh \\ Physical Education Laboratory, Chung-Ang University, \#1419, bldg. 303, Chung-Ang Univ., 84, Heukseok-ro, Dongjak-gu, Seoul, South Korea \\ E-mail: ohpapsy@gmail.com
}

Received: 25-02-2016

Accepted: 26-03-2016

Published: 30-04-2016

doi:10.7575/aiac.ijkss.v.4n.2p.1

URL: http://dx.doi.org/10.7575/aiac.ijkss.v.4n.2p.1

This work was supported by the National Research Foundation of Korea Grant funded by the Korean Government (NRF-2014S1A5B8043862).

\begin{abstract}
Background: Teaching physical education is an emotion-laden context which requires physical education teachers to engage in emotional labor in order to foster their well-being, as well as student's outcomes. Objective: The purpose of this study was to investigate the predictability of emotional labour strategies on job satisfaction and emotional exhaustion among secondary physical education teachers in South Korea. Specifically, the four forms of emotional labour (i.e., surface acting, deep acting, genuine positive expression, and genuine negative expression) were hypothesized to have different influences on job satisfaction and emotional exhaustion. Method: A total of 225 fulltime physical education teachers were invited to participate in the paper-pencil survey. The questionnaires contained items measuring the four forms of emotional labour, emotional exhaustion, and job satisfaction which had been modified to fit the physical education setting. Results: The results indicated that surface acting, genuine positive expression, and genuine expression was significantly associated with emotional exhaustion whereas only genuine positive expression was significantly associated with job satisfaction and emotional exhaustion. Finally, emotional exhaustion mediates the relationship between surface acting and job satisfaction, genuine positive expression and job satisfaction, and genuine negative expression and job satisfaction. Conclusion: These results suggest that emotional labour plays a critical role on physical education teachers' well-being and job attitude.
\end{abstract}

Keywords: emotional regulation, physical education teacher, genuine expression, Asian culture, surface acting

\section{Introduction}

\subsection{Introduce the problem}

Teaching is a highly emotional practice (Sutton, 2005) in that teachers often face emotionally challenging incidents every day when dealing with difficult and hostile students, medical emergencies, or angry parents (Tuxford \& Bradley, 2015). These emotional challenges have been found to influence teachers' well-being, mental health, and job attitude, as well as students' learning outcomes (Chan, 2006). Thus, it is critical for teachers to identify how to cope with emotional demands. Emotional labour, a term first coined by Hochschild (1983), is defined as the process of both regulating one's inner feelings and expressing appropriate emotions at work (Grandey, 2000). Teachers need to purposefully modify and display appropriate emotions in their interaction with students as it has the potential to strengthen their ability to deal with emotional challenges and teaching effectiveness (Sutton, 2005). Emotional labour can be a crucial construct in the physical education (PE) domain as it could identify an effective coping strategy to enhance occupational well-being (e.g., less job burnout and greater job satisfaction) and organisational outcomes (e.g., teaching effectiveness and teacher-student relationship). The significance of the construct can be even greater when we consider that the unique physical structure of the PE context (e.g., a large open space) could elicit a higher degree of emotional challenges (Koustelios \& Tsigilis, 2005). In fact, displaying positive emotions in PE context were found to provide multiple benefits for PE teachers and students (see Stuhr, Sutherland, \& Ward, 2012). For example, in their in-depth interview with two PE teachers, Stuhr and his colleagues (2012) found that displaying positive emotion affected student performance by creating more teacher-student relationship and increasing student's participation. However, few studies have examined what would cost to their well-being during their endeavor to modify inner feelings to display positive emotion (i.e., emotional labour). 
Further, despite the growing concern about emotional labour in teaching, most emotional labour studies tended to focus on Western cultures (e.g., Barber, Grawitch, Carson, \& Tsouloupas, 2011; Keller, Chang, Becker, Goetz, \& Frenzel, 2014; Taxer \& Frenzel, 2015; Tsouloupas, Carson, Matthews, Grawitch, \& Barber, 2010), which implies that there has been relatively little attention to Asian cultures. It is possible that the findings may differ from Asian cultures because of differences in culture and school norms. For example, Korean students and teachers tended to perceive fewer unexpected events, probably because of the holistic reasoning common in East Asia, and thus experienced fewer fluctuations in their emotional status (Choi \& Nisbett, 2000). Thus, it may be meaningful to examine the relationship between Asian PE teachers' emotional labour strategies and its consequences.

\subsection{Changes in Teacher-Student Relationship in the Korean Educational Context.}

Education in South Korea is viewed as a critical element for one's success; there is an old proverb saying, "Students should not step over the teacher's shadow". It means teaching is honored as one of the most glorious professions and students need to respect their teachers. The proverb is firmly rooted in the long Confucian tradition inherent in Korean society, which has shaped the strong hierarchical relationship between elders and young people. In fact, the Korean society considers that a hierarchical structure is the best way to achieve organisational goals effectively. Thus, older people or people in higher positions are typically expected to take responsibility for managing young people or people in lower positions, whereas lower-status people have to obey the higher status people (Lee, Fraser, \& Fisher, 2003). It is not surprising that this is also reflected in the school system, which is 'hierarchical' and 'monotonous'. That is, teachers hold a higher position and even the same authority as parents, while students, who hold a relatively low position, should obey them (Lee, 2007).

However, this situation recently has faced dramatic change, as students increasingly consider themselves recipients of educational services and the majority of parents have become dissatisfied with the quality of education (Kim, 2003). As a result, the hierarchical relationship between teachers and students and teacher's authority has weakened. Consequently, students have begun to challenge teachers' authority, which has led teachers to experience negative emotions and job burnout (Park \& Lee, 2012). In a recent national survey, approximately two-thirds of Korean teachers felt that they were treated by students and their parents in a disrespectful manner, which in turn led to lower job satisfaction and increased turnover intention in the future (Korean Federation of Teachers Association, 2012).

Further, Korean government has recently implemented a new sport policy which led PE teachers to exhibit a higher burnout level than those participants in the past (Ha, King, \& Naegar, 2011). The rapid and dramatic changes in their authority and working environment caused them to experience increased unpleasant emotions in their daily work. Thus, a greater need exists for Korean teachers to engage in emotional labour to regulate negative emotions and behaviors in a disciplinary situation than there has been in past decades. Although the changes in teacher-student dynamics can be witnessed almost everywhere in the world, such changes are more dramatic in Korea compared to other cultures because Korean culture traditionally followed Confucian hierarchy in all aspects of life.

\subsection{Review of Literature}

\subsubsection{Grandey and Gabriel's conceptual framework of emotional labour}

Grandey and Gabriel (2015) have recently presented a conceptual framework of emotional labour consisting of antecedents, consequences, and moderators. According to the framework, emotional labour is the combination of emotion requirements of the situation, the one's internal regulation process, and outward emotion performance. That is, these three components are working together to constitute the overall emotional labour construct (Lee \& Chelladurai, 2015). Specifically, surface acting and deep acting were identified as different types of internal regulation process. The framework also identified individual characteristics (e.g., personality, motivation, and emotional ability) and situational characteristics (e.g., aroused emotions, customer reaction) as antecedents. Employee well-being (job satisfaction and burnout/health) and organisational well-being (interpersonal relationship performance, job performance) were identified as consequences of emotional labour. Finally, several relational factors (e.g., emotional intelligence, identification, and relationship quality) and contextual factors (e.g., job autonomy, financial compensation, and perceived organizational and supervisor support) were included as the moderating factor in the relationship between emotional labour and the proposed consequences.

Emotional requirements of the situations are known as display rules (Hochschild, 1983) which serve as the standards that guide appropriate emotional display. For example, service employees typically follow positive display rules (e.g., kindness and enthusiasm) to please their customers, whereas it is recommended for bill collectors to display negative emotions (e.g., anger) to be effective on their jobs. In the teaching context, it is expected that teachers need to follow two distinctive display rules, which makes the process of emotional labour more difficult. That is, teachers need to determine judiciously which emotions to display at given situations, as sometime they need to display positive emotion (supportive display rules) to encourage students, whereas they should express negative emotion (disciplinary display rules) to discipline students (Barber et al., 2011).

Surface acting involves the process of modifying only outward expressions regardless of their true feelings, whereas deep acting involves the process of modifying "actual feelings" to display appropriate emotions. Thus, individuals who utilize surface acting essentially "fake" the appropriate emotional display, whereas those who utilize deep acting try to genuinely experienced the desired emotions and display them accordingly (Grandey, 2000). Ashforth and Humphrey (1993) introduced genuine expression as the third category of emotional labour, because they considered the previous 
two categories to be insufficient to represent the breadth of the emotional labour process at work. They argued that employees could feel the desired emotions and display them accordingly in automatic ways. For example, teachers may truly feel and express enthusiasm toward their subject matter, and the expression of such enthusiasm is different from deep acting because it requires no modification process. Regulating emotions, albeit automatically, requires labour in order to be consistent with situational requirements. That is, displaying appropriate emotions in response to organisational requirements in a given situation is considered labour, regardless of whether or not one regulates one's emotions spontaneously (Diefendorff, Croyle, \& Gosserand, 2005). Later, Discrete Emotions Emotional Labour Scale (DEELS) was developed by Glomb and Tews (2004), in which genuine expression was divided into the two subcategories - genuine positive expression and genuine negative expression - in order to understand emotional labour in more comprehensive way.

\subsubsection{Emotional Labour and Emotional Exhaustion}

Emotional exhaustion, defined as a chronic fatigue state of emotional depletion (Maslach \& Jackson, 1986), is considered the "central quality of burnout and the most obvious manifestation of this complex syndrome" (Maslach, Schaufeli, \& Leiter, 2001; p. 402). Teaching was found to be highly vulnerable to emotional exhaustion because it involves a high degree of interpersonal dimension (Sutton, 2005). Ample studies have shown that emotionally exhausted teachers experienced decreased job satisfaction and quality of teaching, which in turn negatively influenced students' academic achievement (Blandford, 2000; Ha et al., 2011).

Researchers have frequently found a positive relationship between surface acting and emotional exhaustion among teachers (Barber et al., 2011; Chang, 2009; Taxer \& Frenzel, 2015; Tsouloupas et al., 2010). Individuals utilizing surface acting are required to devote significant psychological effort in the process of suppressing their true feelings. This effort, along with the psychological strain of emotional dissonance, depletes one's emotional resources, which in turn lead to emotional exhaustion (Hochschild, 1983). However, compared to surface acting, deep acting does not generate emotional dissonance but instead elicits a feeling of authenticity, because it entails a match between a felt emotion and a displayed emotion (Brotheridge \& Grandey, 2002).

Unlike surface acting and deep acting, there is little evidence regarding the effect of genuine positive expression and genuine negative expression on emotional exhaustion. Taxer and Frenzel (2015) found negative association between genuine positive expression and emotional exhaustion. Based on such scarce empirical evidence, further exploration is warranted. We hypothesized that PE teachers' genuine positive expression would be negatively associated with emotional exhaustion because it does not generate emotional dissonance and psychological effort. Finally, regarding the relationship between genuine negative expression and emotional exhaustion, Taxer and Frenzel (2015) found a significant positive association between American teachers' genuine negative expression and emotional exhaustion. A number of scholars in health psychology and medical science have found that frequent expressions of negative emotions (e.g., anger and sadness) had a negative impact on individual health, and were associated with a range of chronic illnesses, from cardiovascular disease to diabetes and asthma (Haukkala, Konttinen, Laatikainen, Kawachi, \& Uutela, 2010). Accordingly we posit that

H1: Surface acting will be positively associated with emotional exhaustion.

H2: Deep acting will be negatively associated with emotional exhaustion.

H3: Genuine positive expression will be negatively associated with emotional exhaustion.

H4: Genuine negative expression will be positively associated with emotional exhaustion.

1.3.3 Emotional Labour and Job Satisfaction

Job satisfaction, defined as "a pleasurable or positive mental state resulting from the appraisal of one's job or job experience" (Locke, 1976, p. 1300), is included in this study. Although research on job satisfaction among PE teachers has received limited attention, it has been considered a critical element in teaching due to its association with organizational outcomes including productivity and profit, turnover intention, and actual turnover behavior (see Koustelios \& Tsigilis, 2005). Empirical evidence has shown inconsistent results in the relationship between different types of emotional labour and job satisfaction for teachers (Taxer \& Frenzel, 2015; Yin, 2015; Zhang \& Zhu, 2008). For example, Zhang and Zhu (2008) found that surface acting significantly predicted job satisfaction negatively, whereas other studies found no association between them (Taxer \& Frenzel, 2015; Yin, 2015). Further, Zhang and Zhu (2008) and Yin (2015) found a positive association between deep acting and job satisfaction, whereas Taxer and Frenzel (2015) found no association. Based on such scarce empirical evidence, further exploration is warranted.

Further, it has been found that teachers' genuine positive expression, which theoretically requires no psychological effort and generates no emotional dissonance, was positively associated with job satisfaction, as it is an automatic process (e.g., Taxer \& Frenzel, 2015; Yin, 2015). On the other hand, previous literature has also found that frequent expressions of anger have a negative impact on individuals' job attitudes, because those inner negative emotions entail unpleasant feelings (Baumeister \& Exline, 2000), which in turn result in decreased job satisfaction (Taxer \& Frenzel, 2015). Accordingly, we posit that among Korean PE teachers,

H5: Surface acting will be negatively associated with job satisfaction.

H6: Deep acting will be positively associated with job satisfaction.

H7: Genuine positive expression will be positively associated with job satisfaction.

H8: Genuine negative expression will be negatively associated with job satisfaction. 
Based on the previous evidence regarding the relationship between emotional labour and teacher well-being outcomes, we expected that the association between the four forms of emotional labour and job satisfaction would be mediated by emotional exhaustion. Previous studies have shown that emotional exhaustion is a negative circumstance, and teachers who experience emotional exhaustion are less likely to have job satisfaction (Ha, et al., 2011; Koustelios \& Tsigilis, 2005). For example, Ha and his colleagues (2011) found that job burnout negatively affected job satisfaction and with emotional exhaustion contributing the most compared to other components of burnout among Korean PE teachers. Thus, this study attempted to test whether emotional exhaustion mediates the relations between the four forms of emotional labour and job satisfaction. Accordingly, we posit that among Korean PE teachers

H9: Emotional exhaustion will mediate the relationship between surface acting and job satisfaction.

H10: Emotional exhaustion will mediate the relationship between deep acting and job satisfaction.

H11: Emotional exhaustion will mediate the relationship between genuine positive expression and job satisfaction.

H12: Emotional exhaustion will mediate the relationship between genuine negative expression and job satisfaction.

\subsection{Purpose of the Study}

In summary, this study tested a part of Graney and Gabriel's (2015) conceptual framework by conducting an empirical examination of the relationship among the four forms of emotional labour, emotional exhaustion, and job satisfaction, and the mediating effect of emotional exhaustion among Korean physical education teachers. It is posited that different forms of emotional labor is associated with emotional exhaustion and job satisfaction. More specifically, the purpose of this study was three-fold: (a) to examine the relationship between the four forms of emotional labour and emotional exhaustion; (b) to examine the relationship between the four forms of emotional labour and job satisfaction; and (c) to examine the mediating role of emotional exhaustion in the relationship between the four forms of emotional labour and job satisfaction.

Further, Stuhr and his colleagues (2011) suggested that future PE research need to examine the role of positive emotions and "deep acting (p. 179)" to expand the physical education field. Emotional labour is the recent construct which can shed light on understanding how to intentionally generate and display positive emotions while eliminating the accompanying harmful effect derived by such process. Although the role of positive emotions have been studied in sport science, emotional labor has received relatively little attention in either kinesiology or Asian context. Thus, this study will contribute to kinesiology and emotional labour literature by introducing an unexplored construct of emotional labour and its predictability for Asian cultures.

\section{Methods}

\subsection{Participants}

225 full-time PE teachers from 12 different middle schools in Seoul and vicinity were invited to participate in the study. This study chose a convenience sampling method in order to obtain sufficient number of participants for statistical analysis. Among the total participants, 161 (71.9\%) teachers were male and $63(28.1 \%)$ teachers were female. According to Ministry of Culture, Sports, and Tourism (2014), the total number of middle school PE teachers in Korea are 8,304 in 2013 . They reported that among them, 6,749 (81.2\%) were males and 1,555 (18.8\%) were. Their mean age was $32.40(S D=8.75)$, ranging from 20 to 62 . Job tenure as a PE teacher ranged between 1 and 34 years with a mean value of 12.33 years.

\subsection{Procedure}

Before the main study, researchers conducted a pilot study with 10 PE teachers to establish the validity and the reliability of the questionnaires. Participants were asked to complete the questionnaires and based on their comments and feedbacks, the survey items were modified to improve the validity of the survey. For the main study, two trained research assistants visiting the schools explained the purpose of the study, conducted the face-to-face survey, and gathered the data. The research obtained the approval from the Human Subjects Institutional Review Board at the university in order to protect human subjects.

\subsection{Instrument}

\subsubsection{Surface acing and deep acting.}

The surface acting and deep acting were measured by Brotheridge and Lee's (2003) Emotional Labor Scale (ELS). The four items of surface acting and four items of deep acting were anchored with five-point Likert scale ranged from 1 (never) to 5 (always). The original items were modified so that the items could be applicable to PE teachers and PE teaching context. In employing the scale, participants were asked to respond to the stem "On an average day at schools and PE classes, how often do you do each of the following when both teaching and interacting with students?" Sample items for surface acting are "Pretend to have emotions that I don't really have". Sample items for deep acting are "Really try to feel the emotions I have to show as part of my job". The scale showed an acceptable internal reliability alpha of .76 for surface acting and .90 for deep acting (Brotheridge \& Lee, 2002). 


\subsubsection{Discrete emotion emotional labour scale.}

Regarding genuine positive expression and genuine negative expression, a shorter version of Discrete Emotion Emotional Labor Scale (DEELS, Glomb \& Tews, 2004) was utilized. Four items of discrete positive emotions (i.e., contentment, enthusiasm, liking, and happiness) and four items of negative emotions (e.g., anger, anxiety, frustration, and sadness) were adapted from DEELS. Although the original subscale includes 5 items of discrete positive emotions (i.e., liking) and 10 items of negative emotions (i.e., disliking, aggravation, fear, irritation, distress, hate), panels of the study consisted of one sport pedagogy professor, one sport management professor, and two experienced PE teachers concluded that some of the items were not applicable to teaching context. Additionally, negative emotions were chosen based on typical unpleasant emotions in teaching listed by Chang (2009). A sample item asked "How often do you genuinely express enthusiasm when you feel that way?" Respondents can choose from 1 (I never genuinely express this) to 5 (I genuinely express this many times a day). The scale showed an acceptable internal reliability alpha of .84 for genuine positive expression and .87 for genuine negative expression (Mahoney et al., 2011).

\subsubsection{Emotional exhaustion.}

This study utilized the shorter version of emotional exhaustion subscale of the Maslach Burnout Inventory (Maslach \& Jackson, 1986). A sample item is "I feel used up at the end of the day". The words "recipient" and "people" were replaced by "students", whereas "work" was replaced by "teaching". In previous study, the scale showed a good internal reliability alpha of .83 with the sample of teachers (Barber et al., 2011).

\subsubsection{Job satisfaction.}

PE teacher's job satisfaction was measured by the modified version of Job Satisfaction Subscale of Michigan Organisational Assessment Questionnaire (Cammann, Fichman, Jenkins, \& Klesh, 1979). A higher mean score indicates higher satisfaction with the job. A sample item is "My job is enjoyable". The reliability of the items was Cronbach alpha of .85 in Ha, King, and Naegar's (2011) study with the sample of Korean secondary PE teachers.

\subsection{Data Analyses}

The descriptive statistics were calculated for the research variables along with their reliability estimation (i.e., Cronbach's alpha) using SPSS 21.0. To satisfy the basic assumption of structural equation modeling (SEM), normality of the data were checked using skewness and kurtosis. After the normality of the data was examined, both measurement models and structural models were tested using AMOS 21.0. A confirmatory factor analysis (CFA) was conducted on the latent variables in order to check convergent and discriminant validity of the variables. The researchers assessed structural coefficients of the relationship in order to test the proposed structural relationship. Regarding mediation analysis, Iacobucci, Saldanhan and Deng (2007) has argued that structural equation analyses has a great advantage to test mediation hypotheses compared to comparing a series of different models. Thus, the researchers utilized structural equation modeling to test mediation analysis in that both direct and indirect relations were tested simultaneously. Specifically, a bootstrapping procedure (using 1,000 re-samples) were performed to test the whether there were significant mediating effects in the proposed relationship. The researcher used the well-established maximum likelihood estimation such as $\chi^{2} / d f$ ratio, CFI, TLI, and NFI to evaluate the fit of the model. Kline (2005) suggested that $\chi^{2} / d f$ ratio less than 2.0 was considered good. For NFI, CFI and TLI, values higher than .90 are considered to have an acceptable and values greater than .95 indicate a good fit to the data. In addition, RMSEA and SRMR values less than .06 indicates a close fit and values less than .08 indicates a reasonable fit of a model (Hu \& Bentler, 1999).

\section{Results}

\subsection{Descriptive Statistics, Reliability, and Validity}

Descriptive statistics including mean scores, standard deviation, skewness, and kurtosis were calculated to detect any outliers and invalid data. A total of 225 responses were utilized for further data analyses. Alpha coefficients, descriptive statistics (means, standard deviations, skewness, and kurtosis) and correlation coefficients for all variables are presented in Table 1.

Table 1. Descriptive Statistics and Correlations among Study Variables

\begin{tabular}{lcccccc}
\hline & 1 & 2 & 3 & 4 & 5 & 6 \\
\hline 1. Surface Acting & $(.72)$ & & & & & \\
2. Deep Acting & $.14^{* *}$ & $(.86)$ & & & & \\
3. Genuine Positive Expression & -.11 & $.19^{* *}$ & $(.90)$ & & & \\
4. Genuine Negative Expression & $.30^{* *}$ & -.08 & .12 & $(.69)$ & & \\
5. Emotional Exhaustion & $.19^{* *}$ & $-.15^{* *}$ & -.09 & $.28^{* *}$ & $(.79)$ & \\
6. Job Satisfaction & -.01 & $.24 * *$ & $.33^{* *}$ & $-.20^{* *}$ & $-.44^{* *}$ & $(.84)$ \\
Mean & 3.38 & 3.73 & 3.47 & 1.74 & 2.29 & 4.01 \\
Standard deviation & .67 & .64 & .89 & .51 & .67 & .71 \\
Skewness & -.45 & -.90 & -.17 & 1.19 & .26 & -.48 \\
Kurtosis & .23 & 1.77 & -.76 & 2.54 & -.02 & .13 \\
\hline
\end{tabular}

Note. $\alpha$ coefficient on the diagonal. Bivariate correlations among the study variables are significant at ${ }^{*} p<.05 * * p<$ .01 


\subsection{Confirmatory Factor Analysis}

Then, the convergent and discriminant validity of all constructs were tested using CFA. Results of confirmatory factor analysis showed a good model fit, $\chi^{2} / d f=160.78 / 98=1.64, p<.05$; RMSEA $(90 \% \mathrm{CI})=.053(.038-.068)$; SRMR $=$ $.043 ; \mathrm{NFI}=.95 ; \mathrm{TLI}=.94 ; \mathrm{CFI}=.95$. Regarding individual contributions of items to their assigned factors, the items all defined the latent variables well ranged from .45 to .91 , the cut-off point suggested by Stevens (1996). Further, discriminant validity of the constructs were satisfied as the average variance extracted for two constructs exceeded the square of the correlation between the constructs as suggested by Fornell and Larcker (1981).

\subsection{Structural Equation Modeling}

Finally, all hypotheses (as shown in Figure 1.) were tested using structural equation modeling. Overall, the goodness-offit statistics indicated that the structural model showed a good fit, $\chi^{2} / d f=442.76 / 243=1.82$; RMSEA $(90 \%$ CI $)=.061$ $(.052-.069) ;$ SRMR $=.063 ; \mathrm{NFI}=.94 ; \mathrm{TLI}=.94, \mathrm{CFI}=.95$. Regarding the relationship with emotional exhaustion, the results shown in figure 1 indicate that surface acting $(\beta=.24 ; p<.001)$, genuine positive expression, $(\beta=-.16 ; p=.004)$ and genuine negative expression $(\beta=.37 ; p<.001)$ were directly associated with emotional exhaustion, whereas deep acting $(\beta=-.05 ; p>.05)$ were not associated with emotional exhaustion. Thus, H1, H3, and H4 were supported, whereas $\mathrm{H} 2$ was not supported. Further, it was found that only genuine positive expression $(\beta=.22 ; p<.001)$ had a significant direct relationship with job satisfaction whereas neither surface acting $(\beta=.05 ; p>.05)$, deep acting $(\beta=.10 ; p>.05)$, nor genuine negative expression $(\beta=-.06 ; p>.05)$ were directly associated with job satisfaction. Thus, H7 were supported, whereas H5, H6, and H8 were not supported.

\subsection{Mediation Analysis}

H9 to H12 suggests that the relationship between the four forms of emotional labour and job satisfaction is mediated by emotional exhaustion. Figure 1 shows that direct path between surface acting and job satisfaction was not significant. However, if both paths from the independent variable to the mediator and from the mediator to the dependent variable are significant, we can confirm the mediation relationship. In this study, both paths were significant, $\beta_{\text {surface acting } \rightarrow \text { emotional }}$

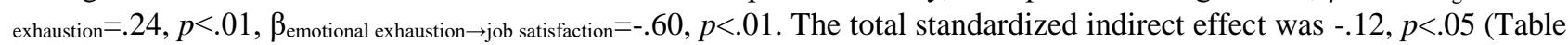
2). Additionally, the mediation path from genuine negative expression to job satisfaction was also significant in that $\beta_{\text {genuine negative expression } \rightarrow \text { emotional exhaustion }}=.37, p<.01, \beta_{\text {emotional exhaustion } \rightarrow \text { job satisfaction }=-.60, p<.01 \text {. The total standardized indirect }}$ effect was $-.22, p<.01$ (see Table 2). Thus, we can conclude that the relationship between surface acting and job satisfaction, and genuine negative expression and job satisfaction was fully mediated by emotional exhaustion. Finally, the direct path from genuine positive expression to emotional exhaustion and job satisfaction were both significant in

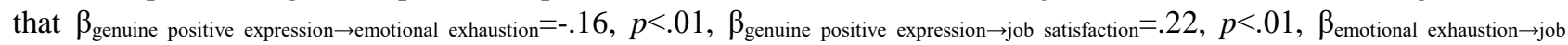
satisfaction=-.60, $p<.01$. Thus, the relationship between genuine positive expression and job satisfaction was partially mediated by emotional exhaustion. Therefore, H9, H11, and $\mathrm{H} 12$ were supported.

Table 2. Mediation analysis

\begin{tabular}{cccc}
\hline Variable & direct effect $\beta$ & indirect effect $\beta$ & BC 95\% Confidence Interval \\
\hline SA & Not significant & $-.12^{* *}$ & -.25 to -.01 \\
\hline GPE & $.22^{* *}$ & $.18^{* *}$ & .05 to .31 \\
\hline GNE & Not significant & $-.22^{* *}$ & -.39 to -.10 \\
\hline $\mathrm{EE}$ & $-.60^{* *}$ & $\mathrm{n} / \mathrm{a}$ & $\mathrm{n} / \mathrm{a}$ \\
\hline
\end{tabular}

Note. $\beta$ values are standardized coefficients. Dependent variable is job satisfaction. $* p<.05 * * p<.01$

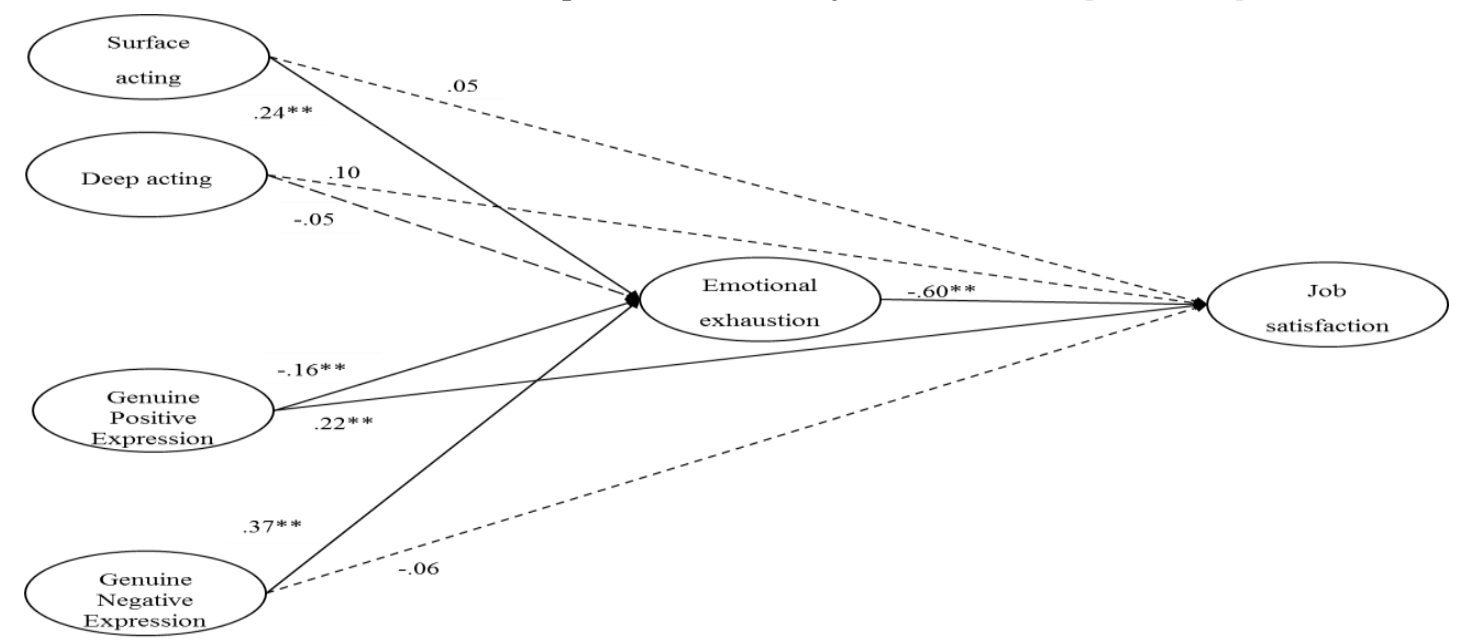

Figure 1. Structural equation model showing the mediating relationship among surface acting, deep acting, genuine positive expression, genuine negative expression, emotional exhaustion, and job satisfaction

Note. Solid lines represent significant relationships; dotted lines represent nonsignificant relationships. $* p<.05 . * * p<.01$ 


\section{Discussion}

This study tested a part of Grandey and Gabriel's (2015) conceptual framework of emotional labour by examining the relationship between the four forms of emotional labour and individual outcomes of job satisfaction and emotional exhaustion, and to test the mediating role of emotional exhaustion in these relationships among Korean PE teachers. The results partially supported the hypotheses of the study, making important theoretical contributions to the literature on emotional labor literature.

Regarding the direct relationship between the four forms of emotional labour and emotional exhaustion, surface acting, genuine positive expression, and genuine negative expression were significantly associated with emotional exhaustion. That is, PE teachers who chose to fake their inner feelings and express either their true positive or negative feelings are either more or less likely to be emotionally exhausted. Unlike deliberate emotional labour strategies (i.e., surface acting and deep acting) which requires psychological efforts, genuine positive expression is an automatic process and does not require efforts and create dissonance (Muraven \& Baumeister, 2000). Thus, those who utilize genuine positive expression will be less likely to experience emotional exhaustion whereas those who engage in surface acting will be more likely to experience emotional exhaustion. This is consistent with the findings of Barber et al. (2011), Chang (2009), Taxer \& Frenzel (2015), Tsoulouspas et al. (2010), and Yin (2015).

In partial support of our hypothesis regarding the direct relationship between the four forms of emotional labour and job satisfaction, only genuine positive expression was significantly associated with job satisfaction. That is, PE teachers who naturally experience and express positive emotions are more likely to satisfy with their jobs. This is consistent with the findings of Taxer \& Frenzel (2015), Yin (2015), and Zhang \& Zhu (2008).

Although this study found no direct relationship between surface acting and job satisfaction, we found that the relationship was fully mediated by emotional exhaustion. As hypothesized, the relationship between emotional exhaustion and job satisfaction was significant (Koustelios \& Tsigilis, 2005). Further, it was found that surface acting became influential, affecting emotional exhaustion even though it had no direct impact on job satisfaction. Thus, our result demonstrated that although surface acting may not affect job dissatisfaction directly, it would decrease PE teachers' satisfaction toward their jobs by making them emotionally exhausted. Similarly, although genuine negative expression had no direct impact on job satisfaction, PE teachers' job satisfaction would be decreased by making them emotionally exhausted.

The current study makes several practical contributions. Our results suggest that PE teachers who suppress their true emotions or display true negative emotions will be emotionally exhausted and be dissatisfied with their job to a significant degree. As a countermeasure, Korean school administrators may need to implement emotional labour training programs in order for PE teachers to avoid a surface acting strategy and genuine negative expression as emotional labour is a skill that can be taught and developed through practice and repetition (Grandey, Fisk, Matila, \& Jansen, \& Steiner, 2005). For example, a program can be designed to compel PE teachers to deliberately experience the situation that generates aversive mood in PE context (through video clip or articles). Then those teachers learn how to cope with the aversive emotions in more appropriate way. Through repetition, the regulating process may become more stable and require less effort from time to time.

Without the intervention program, PE teachers also need to develop their own coping strategies in order to manage negative emotions and express genuine positive emotions intentionally. For example, Stuhr and his colleagues (2012) found that PE teachers can elicit positive emotion climate by active listening, showing concern and encouraging students.

Sutton (2005) also provided relevant emotional regulation strategies for teachers. First, PE teachers can keep an emotion teaching diary to identify common patterns in timing and situations where positive and negative emotions are aroused and. Second, PE teachers can ask students about the consequences of emotional expression during the class and help them identify how different kinds of emotions influence students differently. Third, it is necessary for PE teachers to create an environment that helps them trigger positive emotions and calmness. For example, previous research has suggested that situational stimulus in everyday life has a potential to activate and influence emotional status and behaviors (see Aarts \& Dijksterhuis, 2003). By extension, PE teachers should post certain cues around them to activate their willingness to express appropriate emotions. The cues may include a personalized "Keep Calm and Carry On poster", a picture of a landscape, or soft music that generates peace of mind.

Finally, Sutton (2005) addressed specific strategies to prevent negative emotions from becoming fully developed and to manage negative emotions during the class. Preventive strategies to defuse potential problem situations include stepping back and telling a joke. When negative emotions already have been elicited, PE teachers can use self-talk and reappraise the situation by reminding themselves that disruptive students are kids, or that they may be stressed out due to excessive parental demands. Several behavioral strategies can also help PE teachers deal with negative emotions, such as moving away from the situation or taking a deep breath and not taking students' comments or behavior personally.

\subsection{Limitations and recommendations for future research}

Methodologically, this study was cross-sectional study and used self-reported data from convenience sampling. Which might generate the common method bias. Thus, it should be cautious to infer the causality and directions among variables. That is, it is possible that the direction of the relationship is either the opposite or reciprocal. For example, 
emotional exhaustion may lead PE teachers to perform more surface acting as they depleted most of their psychological resources to engage in a cognitive reappraisal process (i.e., deep acting). Future research may implement an experimental study or a longitudinal study in order to confirm such reciprocal relationships. Additionally, this study used all questionnaires from other domains after the revision process; thus, future studies may need to develop new scales that can represent PE context in more clear way. Further, future studies may utilize a mixed method (Carson, 2009), combining quantitative and qualitative research techniques in order to collect multiple kinds of data, which allows for the "opportunity to compensate for inherent method weaknesses, capitalize on inherent method strengths, and offset inevitable method biases" (Greene, 2007, p. xiii).

Moreover, we focused on only two outcome variables of teacher well-being based on Grandey and Gabriel's (2015) conceptual framework, job satisfaction and emotional exhaustion. Future research should attempt to examine other key variables associated with teaching in terms of antecedents (i.e., affective event, emotional intelligence, affectivity, and personality), teacher well-being (i.e., physical health and organizational commitment), and teaching effectiveness (i.e., the quality of teacher-student relationships, students' perceived organizational climate, students' satisfaction, and students' trust toward teachers). Further, in terms of the effect of emotional labour on individual outcomes, these relationships might be moderated by several variables, including emotional intelligence, perceived social support, job autonomy, financial reward, and the teacher-student relationship quality.

Particularly, emotional intelligence, defined as the individual's ability to perceive, understand, utilize, and manage emotions in the self and others (Mayer \& Salovey, 1997) can be a critical construct which has a particular implication to the study of emotional labour (Grandey \& Gabriel, 2015; Lee, Chelladurai, \& Kim, 2015). According to Opengart (2000), emotional intelligence involves cognitive ability to understand and mange emotions, whereas emotional labour is a behavioral process of expressing emotions outwardly. Thus, high emotional intelligence teachers possibly can perform more effective or highly advanced emotional labour strategies. Future research may need to examine the relationship between emotional intelligence and emotional labour strategies as an antecedent.

\section{Conclusion}

In summary, this study contributes to the both emotional labour and kinesiology literature by testing Grandey and Gabriels' (2015) conceptual framework and examining both direct and indirect relationship between the four forms of emotional labour and the individual outcomes (i.e., emotional exhaustion and job satisfaction). Thus, our research confirms the part of of Grandey and Gabriel's (2015) conceptual framework of emotional labour and identifies emotional exhaustion as an important mediator. Our study shows that PE teachers' emotional labour has significant implications for their individual outcomes. Thus, Korean school administrators need to consider developing an intervention program to decrease emotional exhaustion and enhance job satisfaction for PE teachers; which in turn influence student's learning experience. Further, Korean PE teachers need to understand the benefit of positive emotions during the class while the process might have double-edge effects on their personal well-being. We hope that this study can help PE teachers create a desirable working environment and identify health-beneficial coping strategies in their workplace.

\section{References}

Aarts, H., \& Dijksterhuis, A. (2000). Habits as knowledge structures: Automaticity in goal-directed behavior. Journal of Personality and Social Psychology, 78, 53-63.

Ashforth, B. E., \& Humphrey, R. H. (1993). Emotional labor in service roles: The influence of identity. Academy of Management Review, 18(1), 88-115.

Barber, L., Grawitch, M., Carson, R., Tsouloupas, C. (2011). Costs and benefits of supportive vs. disciplinary emotion regulation strategies in teachers. Stress and Health, 27(3), 173-187.

Baumeister, R. F., \& Exline, J. J. (2000). Self-control, morality, and human strength. Journal of Social and Clinical Psychology, 19, 29-42.

Blandford, S. (2000). Managing Professional Development in Schools. London: Routledge .

Brotheridge, C., \& Grandey, A. (2002). Emotional labor and burnout: Comparing two perspectives of "people work." Journal of Vocational Behavior, 60, 17-39.

Brotheridge, C. M., \& Lee, R. T. (2002). Testing a conservation of resources model of the dynamics of emotional labor. Journal of Occupational Health Psychology, 7(1), 57-67.

Cammann, C., Fichman, M., Jenkins, D., \& Klesh, J. (1979). The Michigan Organizational Assessment Questionnaire. Unpublished manuscript, University of Michigan, Ann Arbor.

Chang, M. (2009). An appraisal perspective of teacher burnout: Examining the emotional work of teachers. Educational Psychology Review, 21, 193-218.

Choi, I., \& Richard, N. (2000). Cultural psychology of surprise: Holistic theories and recognition of contradiction. Journal of Personality and Social Psychology, 79(6), 890-905. 
Diefendorff, J. M., Croyle, M. H., \& Gosserand, R. H. (2005). The dimensionality and antecedents of emotional labor strategies. Journal of Vocational Behavior, 66, 339-357.

Fornell, C., \& Larcker, D. F. (1981). Evaluating structural equation models with unobservable variables and measurement error. Journal of Marketing Research, 18, 39-50.

Glomb, T. M., \& Tews, M. J. (2004). Emotional labor: A conceptualization and scale development. Journal of Vocational Behavior, 64, 1-23.

Grandey, A. A. (2000). Emotion regulation in the workplace: A new way to conceptualize emotional labor. Journal of Occupational Health Psychology, 5, 95-110.

Grandey, A., \& Gabriel, A. (2015). Emotional labor at a crossroads: Where do we go from here? Annual Review of Organizational Psychology and Organizational Behavior, 2, 323-349.

Grandey, A., Fisk, G., Matilla, A., Jansen, K., \& Steiner, D. (2005). Is "service with a smile" enough? Authenticity of positive displays during service encounters. Organizational Behavior and Human Decision Processes, 96(1), 38-55.

Greene, J. (2007). Mixed methods in social inquiry. New York: Wiley.

Ha, J., King, K. M., \& Naeger, D. J. (2011). The impact of burnout on work outcomes among South Korean physical education teachers. Journal of Sport Behavior, 34(4), 343-357.

Haukkala, A., Konttinen, H, Laatikainen, T., Kawachi, I., Uutela, A. (2010). Hostility, anger control, and anger expression as predictors of cardiovascular disease. Psychosomatic Medicine, 72(6), 556-562.

Hochschild, A. R. (1983). The Managed Heart: Commercialization of Human Feeling. Palo Alto, CA: University of California Press.

Hu, L., \& Bentler, P. M (1999). Cutoff criteria for fit indexes in covariance structure analysis: Conventional criteria versus new alternatives. Structural Equation Modeling, 6, 1-55.

Iacobucci, D., Saldanhan, N., \& Deng, X. (2007). A mediation on mediation: Evidence that structural equations models perform better than regression. Journal of Consumer Psychology, 17(2), 139-153.

Keller, M., Chang, M., Becker, E., Goetz, T., \& Frenzel, A. (2014). Teachers' emotional experiences and exhaustion as predictors of emotional labor in the classroom: an experience sampling study. Frontier Psychology, 5, 1-10.

Kim, M. (2003). Teaching and learning in Korean classrooms: The crisis and the new approach. Asia Pacific Education Review, 4, 140-150.

Kline, R. (2005). Principles of Structural Equation Modeling. New York, NY: Guildford Press.

Korean Federation of Teacher's Association. (2012). Teacher's day. http://www.kfta.or.kr/news/view.asp?bName=news\&sdiv=1\& num=4874.

Koustelios, A., \& Tsigilis, N. (2005). The relationship between burnout and job satisfaction among physical education teachers: a multivariate approach. European Physical Education Review, 11(2), 189-203.

Lee, S. (2007). The relations between the student-teacher trust relationship and school success in the case of Korean middle schools. Educational Studies, 33(2), 209-216.

Lee, Y., Chelladurai, P., \& Kim, Y. (2015). Emotional labor in sports coaching: Development of a model. International Journal of Sports Science and Coaching, 10(2-3), 561-575.

Lee, S., Fraser, B., \& Fisher, D. (2003). Teacher-student interactions in Korean high school science classrooms. International Journal of Science and Mathematics Education, 1, 67-85.

Locke. E. A. (1976). The nature and causes of job satisfaction. In M.D. Dunnette (Ed.), Handbook of Industrial and Organizational Psychology, Chicago, IL: Rand McNally.

Locke, E. A. Latham , G. P. (1990). A theory of goal setting and task performance. Englewood Cliffs, NJ: Prentice Hall.

Mahoney, K. T., Buboltz, W. C., Buckner, J. E., \& Doverspike, D. (2011). Emotional labor in American professor. Journal of Occupational Health Psychology, 16(4), 406-423.

Maslach, C., \& Jackson, S. E. (1986). Maslach Burnout Inventory Manual (2nd ed.). Palo Alto, CA: Consulting Psychologists Press.

Maslach, C., Schaufeli, W., \& Leiter, M. (2001). Job burnout. Annual Review of Psychology, 52, 397-422.

Mayer, J., \& Salovey, P. (1997). Emotional development and emotional intelligence: Implications for educators. New York, NY: Basic Books.

Ministry of Culture, Sports, and Tourism. (2014). 2013 Sports White Paper. Seoul: Korean Institute of Sports Science.

Park, Y. M., \& Lee, S. M. (2012). A longitudinal analysis of burnout in middle and high School Korean teachers. Stress and Health, 29(5), 427-431. 
Shin, H., Puig, A., Lee, J., Lee, J., \& Lee, S. (2011). Cultural validation of the Maslach Bunout Inventory for Korean students. Asia Pacific Educational Psychology, 12, 633-639.

Stevens, J. (1996). Applied multivariate statistics for the social sciences (3rd ed.). Mahwah, NJ: Lawrence Erlbaum Associates.

Stuhr, P., Sutherland, S., \& Ward, P. (2011). Lived-positive emotionality in elementary physical education. Pedagogies: An International Journal, 7(2), 165-181.

Sutton, R. (2005). Teachers' emotions and classroom effectiveness. The relevance of Educational Psychology to Teacher Education, 78(5), 229-234.

Taxer, J., \& Frenzel, A. (2015). Facets of teachers' emotional lives: A quantitative investigation of teachers' genuine and hidden emotions. Teaching and Teacher Education, 49, 78-88.

Tsouloupas, C., Carson, R., Matthews, R., \& Barber, L. (2010). Exploring the association between teachers' perceived student misbehavior and emotional exhaustion: the importance of efficacy beliefs and emotion regulation. Educational Psychology, 30(2), 173-189.

Tuxford, L., Bradley, G. (2014). Emotional job demands and emotional exhaustion in teachers. Educational Psychology, 1-19.

Yin, H. (2015). The effect of teachers' emotional labour on teaching satisfaction: moderation of emotional intelligence. Teachers and Teaching: theory and practice, 21(7), 789-810.

Zhang, Q., \& Zhu, W. (2008). Exploring emotion in teaching: Emotional labor, burnout, and satisfaction in Chinese higher education. Communication Education, 57(1), 105-122. 